\title{
Bonding Topologies in Diamondlike Amorphous-Carbon Films
}

\author{
M. P. Siegal, P. N. Provencio, D. R. Tallant and R. L. Simpson \\ Sandia National Laboratories \\ Albuquerque, NM 87185-1421 \\ PREEIVEC \\ FFA 242000 \\ Q.STI \\ B. Kleinsorge and W. I. Milne \\ Department of Engineering, Cambridge University \\ Cambridge CB2 1PZ, UK
}

\begin{abstract}
The carbon ion energy used during filtered cathodic vacuum arc deposition determines the bonding topologies of amorphous-carbon (a-C) films. Regions of relatively low density occur near the substrate/film and film/surface interfaces and their thicknesses increase with increasing deposition energy. The ion subplantation growth results in mass density gradients in the bulk portion of a-C in the growth direction; density decreases with distance from the substrate for films grown using ion energies $<60 \mathrm{eV}$ and increases for films grown using ion energies $>160$ $\mathrm{eV}$. Films grown between these energies are the most diamondlike with relatively uniform bulk density and the highest optical transparencies. Bonding topologies evolve with increasing growth energy consistent with the propagation of subplanted carbon ions inducing a partial transformation of 4-fold to 3-fold coordinated carbon atoms.
\end{abstract}




\section{DISCLAIMER}

This report was prepared as an account of work sponsored by an agency of the United States Government. Neither the United States Government nor any agency thereof, nor any of their employees, make any warranty, express or implied, or assumes any legal liability or responsibility for the accuracy, completeness, or usefulness of any information, apparatus, product, or process disclosed, or represents that its use would not infringe privately owned rights. Reference herein to any specific commercial product, process, or service by trade name, trademark, manufacturer, or otherwise does not necessarily constitute or imply its endorsement, recommendation, or favoring by the United States Government or any agency thereof. The views and opinions of authors expressed herein do not necessarily state or reflect those of the United States Government or any agency thereof. 


\section{DISCLAIMER}

Portions of this document may be illegible in electronic image products. Images are produced from the best available original document. 


\section{Bonding Topologies in Diamondlike Amorphous-Carbon Films}

Amorphous carbon (a-C) films consist of a mixture of 3-fold ( $\mathrm{sp}^{2}$-like) and 4-fold ( $\mathrm{sp}^{3}$-like) coordinated atoms. To first order, the ratio of bond types dictates materials properties. Hydrogen-free amorphous-tetrahedral diamondlike carbon is interesting due to its room temperature growth, high temperature stability near $800{ }^{\circ} \mathrm{C}$, film smoothness, and extreme hardness and optical transparency properties.[1-4] The high concentration of 4-fold coordinated carbon atoms possible in a-C is due to the kinetic energy of the depositing carbon species from physical growth processes such as filtered cathodic vacuum arc (FCVA), pulsed-laser deposition (PLD), and mass-selected ion beam deposition.[1,2,5] Briefly, highly energetic carbon species (10's-to-100's eV) subplant into a growing film several angstroms beneath the surface, creating localized regions of very high pressure extending tens of angstroms in depth. Metastable 4-fold coordinated carbon bonds form in this kinetically-limited environment.[6,7]

We recently used high-resolution transmission electron microscopy (HRTEM) along with Rutherford backscattering spectrometry (RBS) to definitively measure the mass density of a-C films grown by PLD.[8] HRTEM identified distinct layers of different densities within a given film; relative densities were determined using the HRTEM image contrast. In general, every film has an interfacial, bulk, and surface region. The thicknesses of the interfacial and surface regions increase monotonically with deposition energy and are less dense than the bulk layer. We found mass densities as high as $3.35 \mathrm{~g} / \mathrm{cm}^{3}$ for the bulk portion of films grown at our highest PLD energetics, while the densities of the interfacial and surface regions range from $2.3-2.8$ $\mathrm{g} / \mathrm{cm}^{3}$. 


\section{Bonding Topologies in Diamondlike Amorphous-Carbon Films}

Laser ablation of a carbon target results in both neutral and ionic species of various atom cluster sizes with a wide distribution of energies for the growth of a-C films.[9] Films deposited via FCVA use narrower distributions of charge, cluster size and energy.[10] In this letter we describe the evolution of interface and surface density regions, density gradients in the bulk portion of a-C films, and topographical changes in $\mathrm{C}$ bond clustering for films grown by FCVA while varying only the energy of $\mathrm{C}$ ion deposition. We compare these results to those from films grown by PLD to demonstrate the effect of different regimes of kinetic growth.

We grew $55 \mathrm{~nm}$ thick a-C films onto $\mathrm{Si}(100)$ substrates near room temperature using an FCVA system described elsewhere.[11] Briefly, initiating an arc between a graphite cathode and anode generates a carbon plasma. The $\mathrm{C}$ ion species bends through a $90^{\circ}$ curved magnetic solenoid filter to reduce neutrals and particulates. The pressure of the growth chamber rises to $10^{-5}$ Torr during deposition. The $\mathrm{C}$ ion incident energy at the substrate position has a Maxwellian distribution centered near $10 \mathrm{eV}$.[12] Applying dc biases ranging from 0 to $-320 \mathrm{~V}$ to the substrate holder controls the incident ion energy results in average energies of $10-330 \mathrm{eV}$ for $\mathrm{C}$ deposition. We use both HRTEM and Raman spectroscopy (excited from $514.5 \mathrm{~nm}$ light) to analyze the films.

Fig. 1(a-c) shows cross-sectional HRTEM images for a-C films grown using 10, 90, and $330 \mathrm{eV}$ ion energies. The image contrast within the a-C results from variations in mass density. Each film has a distinguishable layer of low density near both the substrate and surface regions, with a region of higher density in the middle, or bulk region. The contrast gradient in the bulk region of the films varies significantly with growth energy. For a-C films grown using $10 \mathrm{eV}$ 


\section{Bonding Topologies in Diamondlike Amorphous-Carbon Films}

ions (fig. 1a), the brightness of the bulk region increases with distance from the substrate, implying decreasing mass density. This density gradient is similar to that reported for growth by PLD and results from the buildup of strain energy within the films. $[8,13]$ For films grown using $90 \mathrm{eV}$ ions (fig. 1b), the density in the bulk portion is very uniform. While use of this higher growth energy overcomes the driving force of the gradient evolution, it also results in film stresses that reduce the critical thickness for delamination below $100 \mathrm{~nm}$. Growth at this energy corresponds to the highest content of 4-fold coordinated carbon.[11] In the film grown using the highest ion energy of $330 \mathrm{eV}$ (fig. 1c), the density behavior is actually reversed, increasing with distance from the substrate.

High magnification images of the a-C/Si(100) interface regions are shown in fig. $1(\mathrm{~d}-\mathrm{f})$ for films grown using 10,90 and $160 \mathrm{eV}$ ions. Two features are noteworthy. First, the thickness of the dark contrast region in the Si substrate just below the interface increases with ion energy. This contrast represents damage in the Si(100) resulting from an accumulation of point defects created by the bombardment of carbon species onto its surface during a-C film growth. The depth of this collateral damage increases with increasing deposition energy. Typical of point defect propagation, the width of the damage region is not homogeneous. Second, the thickness of the light-colored low density interface region of the films increases with increasing growth energy. The same is true for the surface region of the films. The thickness of these two regions are plotted as a function of deposition energy in fig. 2. The existence of these two layers is a direct result of the subplantation growth mechanism for a-C films.[8] The interface region has low density due to the partial backscatter of $\mathrm{C}$ species impinging upon the heavier $\mathrm{Si}$ atoms. Film density cannot increase until the film thickness is greater than the penetration depth of the 


\section{Bonding Topologies in Diamondlike Amorphous-Carbon Films}

impinging carbon ions, which clearly increases with increasing growth energy. The surface region also has lower density than the bulk of a film since it is not overlaid by more carbon and then subplanted to generate a subsurface region of localized high pressure. Again, the thickness of the surface layer increases with increasing ion energy as a direct result of the penetration depth of carbon ions into a carbon surface, and to an even greater extent, the depth of propagation of $\pi$-bonding between carbon atoms resulting from the localized strain of carbon ion subplantation.

Raman spectroscopy can investigate the nature of the $\pi$-bonded species; spectra for each film are shown in fig. 3. The flat-topped feature occurring between 925 and $1025 \mathrm{~cm}^{-1}$ is a second-order phonon band from the Si substrate. Since the films are nominally the same thickness, the intensity of Si band is indicative of the optical transparency of the a-C. The broad band between 1050 and $1800 \mathrm{~cm}^{-1}$ and centered nominally at $1550 \mathrm{~cm}^{-1}$ is due to resonantlyenhanced vibrational modes of 3-fold coordinated carbon. To first order, the intensity of this band is related to the fraction of 3-fold coordinated carbon. The integrated intensities of both the Si and $C$ peaks for each film are plotted versus deposition energy in fig. 4. Optical transparency increases for films grown using energies up to $60 \mathrm{eV}$ and decreases with the use of higher growth energies. The intensity of the carbon Raman bands has a minimum for films grown using $90 \mathrm{eV}$ ions, which correlates to the highest percentage of 4-fold coordinated carbon atoms measured from electron energy loss spectroscopy.[11] The difference between the deposition energies which optimize transparency and diamondlike bonding is likely a result of the surface and interface layer thicknesses plotted in fig. 2. 


\section{Bonding Topologies in Diamondlike Amorphous-Carbon Films}

The carbon Raman bands are due to vibrational modes involving only the 3-fold coordinated atoms. We deconvolute these spectra in a manner described elsewhere for films grown by PLD.[13] Briefly, we use the physical characteristics of idealized 5-, 6-, and 7membered $\pi$-bonded carbon ring clusters.[14] We do not presume that only ring structures exist, just that they are most likely responsible for the resonant-enhanced Raman signatures from visible excitation wavelengths. At high PLD growth energies, the fraction of 3-fold coordinated atoms is low, resulting in a preponderance of small, isolated clusters of 6-membered rings, which are both thermodynamically stable and planar in geometry. At low PLD growth energies, with a higher 3-fold coordinated C content, such structures are larger and more densely packed, resulting in the incorporation of 5- and 7-membered rings, similar to fullerenes, to impart curvature to their surfaces. Fig. 5 shows the contribution of each ring-related vibrational frequency to the Raman bands shown in fig. 3 for the films grown by FCVA. The ring distribution for the film grown using the lowest FCVA energy appears similar to that for films grown near the highest achievable PLD energies, as does the optical transparency, interface and surface layer thicknesses, and density gradient within the bulk region of the films.[13] Hence, it appears that PLD and FCVA produce a-C films using different regimes of energy with only a small overlap. This likely accounts for the differences found in measured properties of films grown with these two techniques, such as field emission, electrical transport behavior, and the stability of nitrogen-doped films.[15-19]

The trend of increasingly isolated clusters of 6 -membered $\pi$-bonded rings with increasing growth energies continues to near $60 \mathrm{eV}$. Above this energy, the additional contributions to the carbon Raman band are from 5-membered rings, which add curvature and infer the growth of 


\section{Bonding Topologies in Diamondlike Amorphous-Carbon Films}

larger $\pi$-bonded clusters. This correlates with the increasing thicknesses of low density interface and surface regions, decreasing optical transparency, and published results showing increasing fractions of $\pi$-bonded carbon.[11] However, the absence of an increase in the 7-membered ring distribution suggests that the process leading to an increased $\pi$-bonded fraction is driven by thermodynamics, rather than by kinetics; 5 -membered $\pi$-bonded carbon rings are more stable than 7-membered rings.[13] This is consistent with dissipation during high energy $C$ ion bombardment during growth.

Clearly, the transformation of 3- to 4-fold coordinated carbon is more complex than simply subplanting atoms to generate sufficient localized pressure. The energetic arrival of carbon is responsible for both creating and destabilizing 4-fold coordinated bonding. Several factors may result in the destabilization of these bonds. One may simply be the generation of surface thermal energy spikes that result in the formation of the more stable $\pi$-bonds. However, this is unlikely since a-C can be annealed to $800{ }^{\circ} \mathrm{C}$ without significant change in the Raman band shape.[15] Rather, it is more likely that $\pi$-bonding between carbon atoms propagates in a surface well below an impinging $\mathrm{C}$ ion. The energy for converting local electron bonding arrangements is relatively small compared to a large mass transfer of atoms. Conservation of energy would require the depth of $\pi$-bond propagation to scale with the kinetic energy of the impinging carbon species. Such propagation is due to the growth energetics and is responsible for the width of the lower density interface and surface layers, the density gradient within the bulk region, and the evolution of $\pi$-bonded topologies in amorphous carbon films. 


\section{Bonding Topologies in Diamondlike Amorphous-Carbon Films}

The authors thank J. C. Barbour for valuable discussions. Sandia is a multiprogram laboratory operated by Sandia Corporation, a Lockheed Martin Company, for the U.S. DOE under Contract DE-AC04-94AL85000.

\section{References:}

1 P. J. Martin, S. W. Filipczuk, R. P. Netterfield, J. S. Field, D. F. Whitnall, and D. R. McKenzie, J. Mater. Sci. Lett. 7, 410 (1988).

2 S. S. Wagal, E. M. Juengerman, and C. B. Collins, Appl. Phys. Lett. 53, 187 (1988).

3 D. L. Pappas, K. L. Saenger, J. Bruley, W. Krakow, J. J. Cuomo, J. Appl. Phys. 71, 5675 (1992).

4 F. Xiong, Y. Y. Wang, V. Leppert, and R. P. H. Chang, J. Mater. Res. 8, 2265 (1993).

5 S. Kasi, H. Kang, and J. W. Rabalais, Phys. Rev. Lett. 59, 75 (1987).

6 Y. Lifshitz, S. R. Kasi, and J. W. Rabalais, Phys. Rev. Lett. 62, 1290 (1989).

7 D. R. McKenzie, D. Muller, and B. A. Pailthorpe, Phys. Rev. Lett. 67, 773 (1991).

8 M. P. Siegal, J. C. Barbour, P. N. Provencio, D. R. Tallant, and T. A. Friedmann, Appl. Phys. Lett. 73, 759 (1998).

9 A. A. Puretsky, D. B. Geohegan, G. E. Jellison, Jr., and M. M. McGibbon, Appl. Surf. Sci. 96-98, 859 (1996).

10 M. Chhowalla, C. A. Davis, M. Weiler, B. Kleinsorge, and G. A. J. Amaratunga, J. Appl. Phys. 79, 2237 (1996).

11 M. Chhowalla, J. Robertson, C. W. Chen, S. R. P. Silva, C. A. Davis, G. A. J. Amaratunga, and W. I. Milne, J. Appl. Phys. 81, 139 (1997).

12 M. M. M. Bilek, M. Chhowalla, and W. I. Milne, Appl. Phys. Lett. 71, 1777 (1997). 
Bonding Topologies in Diamondlike Amorphous-Carbon Films

13 M. P. Siegal, D. R. Tallant, L. J. Martinez-Miranda, J. C. Barbour, R. L. Simpson, and D. L. Overmyer, Phys. Rev. B (in press).

14 T. E.Doyle and J. R. Dennison, Phys. Rev. B 51, 196 (1995).

15 T. A. Friedmann, K. F. McCarty, J. C. Barbour, M. P. Siegal, D. C. Dibble, Appl. Phys. Lett. 68, 1643 (1996).

16 N. Missert, T. A. Friedmann, J. P. Sullivan, and R. G. Copeland, Appl. Phys. Lett. 70, 1995 (1997).

17 B. S. Satyanarayana, A. Hart, W. I. Milne, and J. Robertson, Appl. Phys. Lett. 71, 1430 (1997).

18 J. P. Sullivan, T. A. Friedmann, R. G. Dunn, E. B. Stechel, P. A. Schultz, M. P. Siegal, and N. Missert, Mater. Res. Soc. Proc. 498, 97 (1998).

19 M. P. Siegal, D. L. Overmyer, D. R. Tallant, J. C. Barbour, R. L. Simpson, N. Conway, and W. I. Milne, (unpublished). 


\section{Figure Captions}

1. (a-c) HRTEM cross-sectional images of a-C films grown using FCVA energies of 10,90 , and $330 \mathrm{eV}$, respectively. (d-f) HRTEM images of the $\mathrm{Si} / \mathrm{a}-\mathrm{C}$ interface for films grown using deposition energies of 10,90 , and $160 \mathrm{eV}$, respectively.

2. Thickness of the surface and interface regions within a-C films grown as a function of deposition energy.

3. Raman spectra for films grown at $10,60,90,160$, and $330 \mathrm{eV}$. The narrow band between 925 and $1025 \mathrm{~cm}^{-1}$ is due to the underlying Si substrate. The broad band between 1050 and $1800 \mathrm{~cm}^{-1}$ is due to resonantly-enhanced vibrational modes of 3-fold coordinated carbon.

4. The integrated intensities of the Si and C Raman bands vs. deposition energy.

5. Relative distribution of Raman vibrations from 5-, 6-, and 7-membered carbon ring structures vs. deposition energy. 

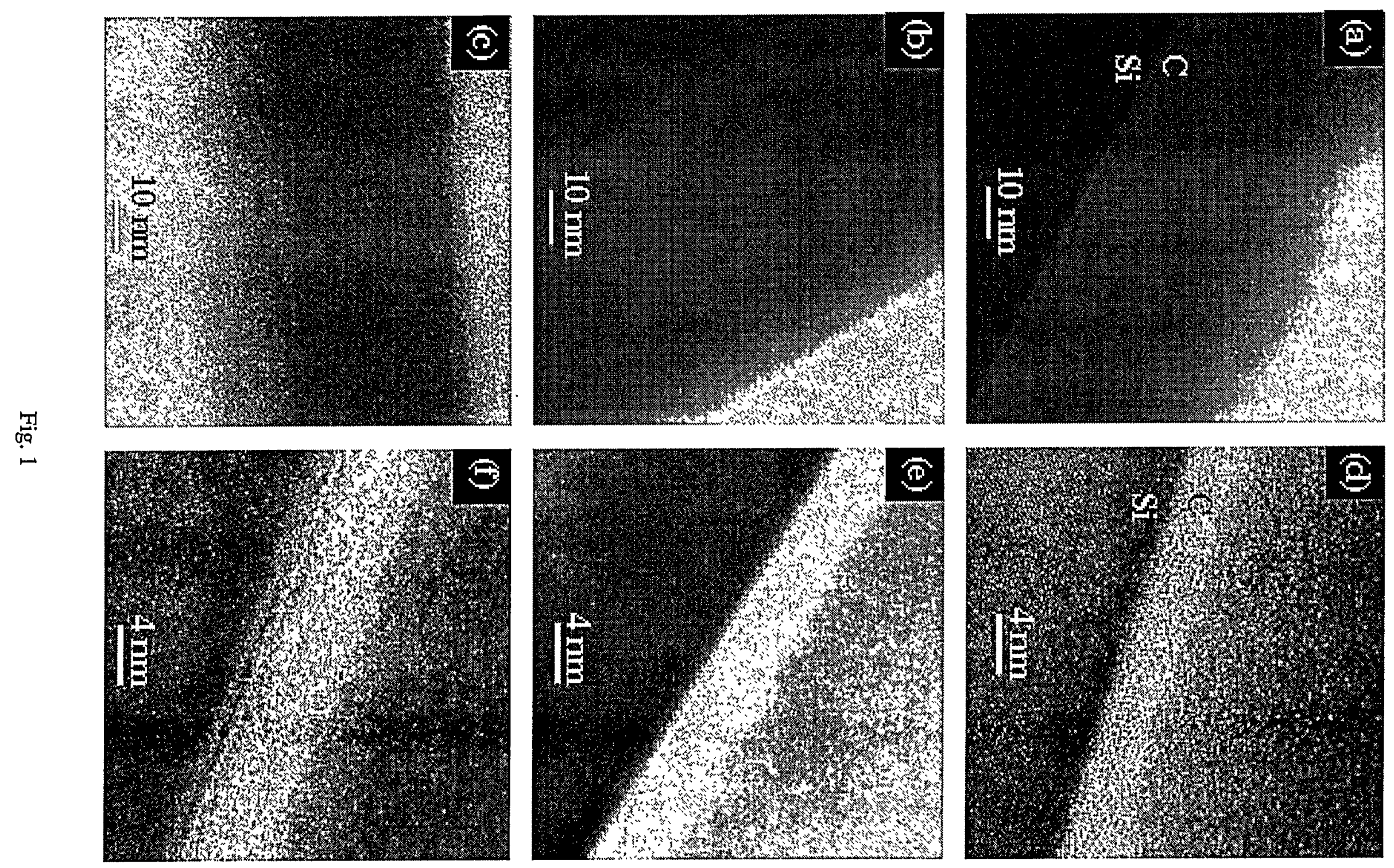


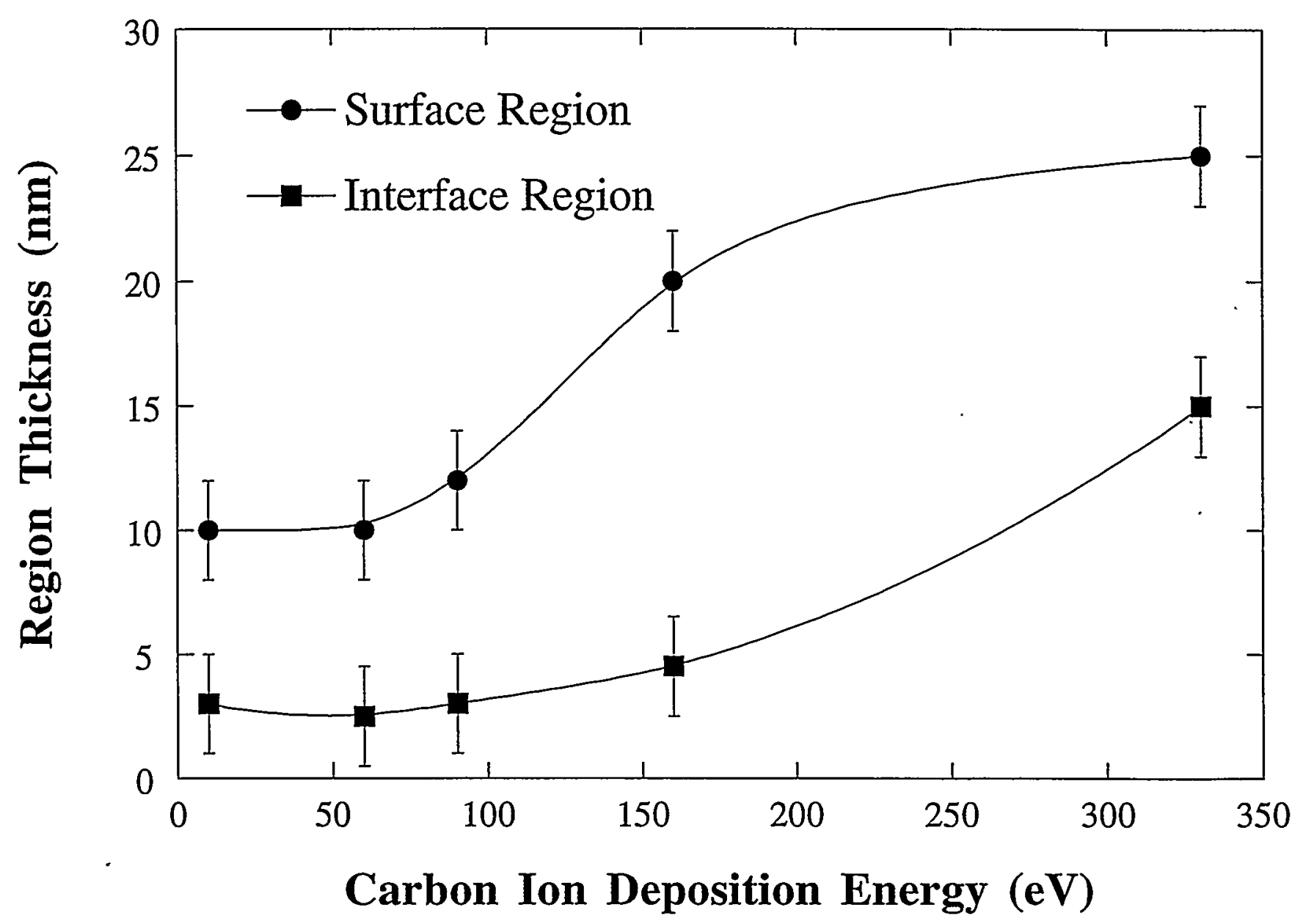

Fig. 2 


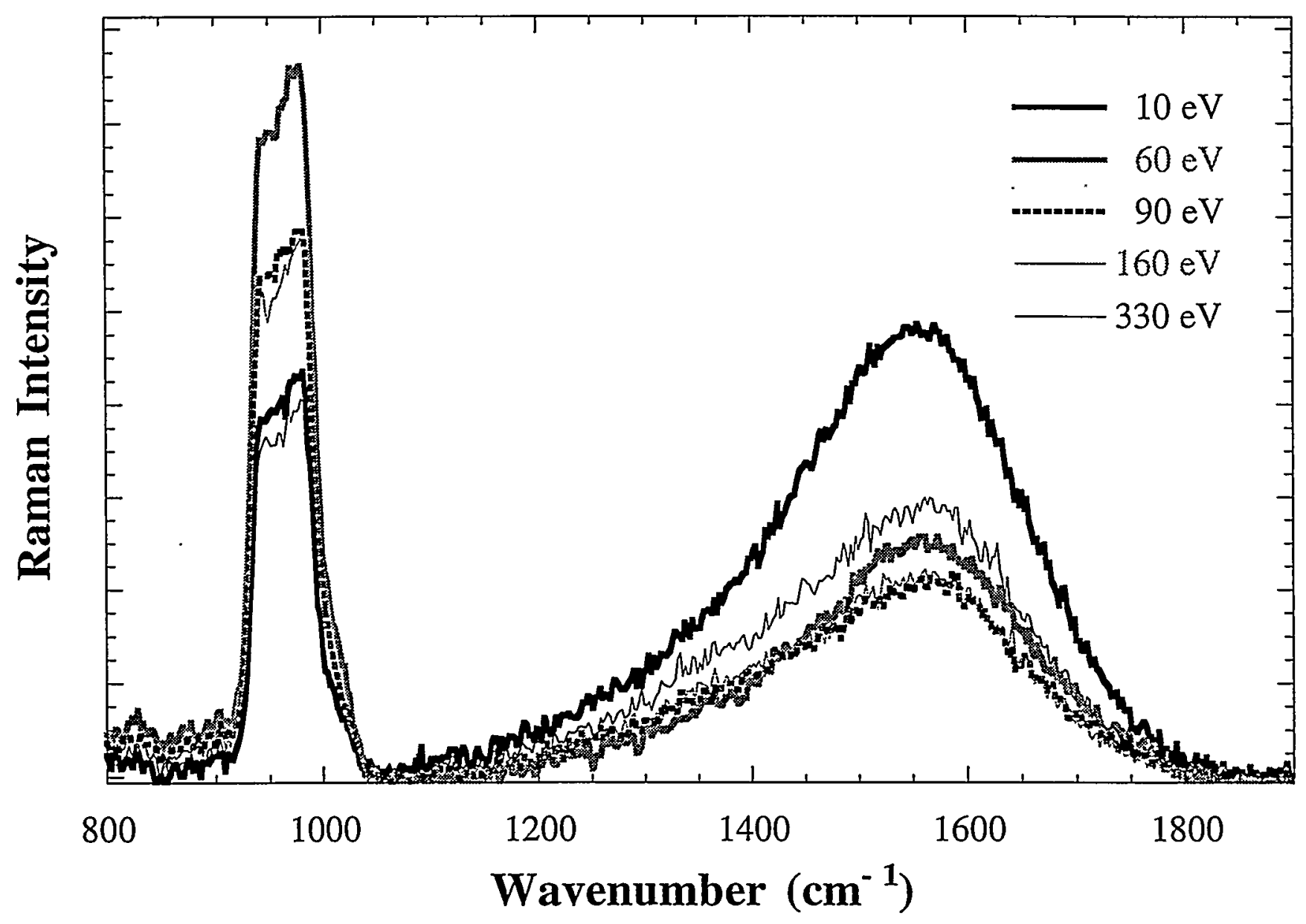

Fig. 3 


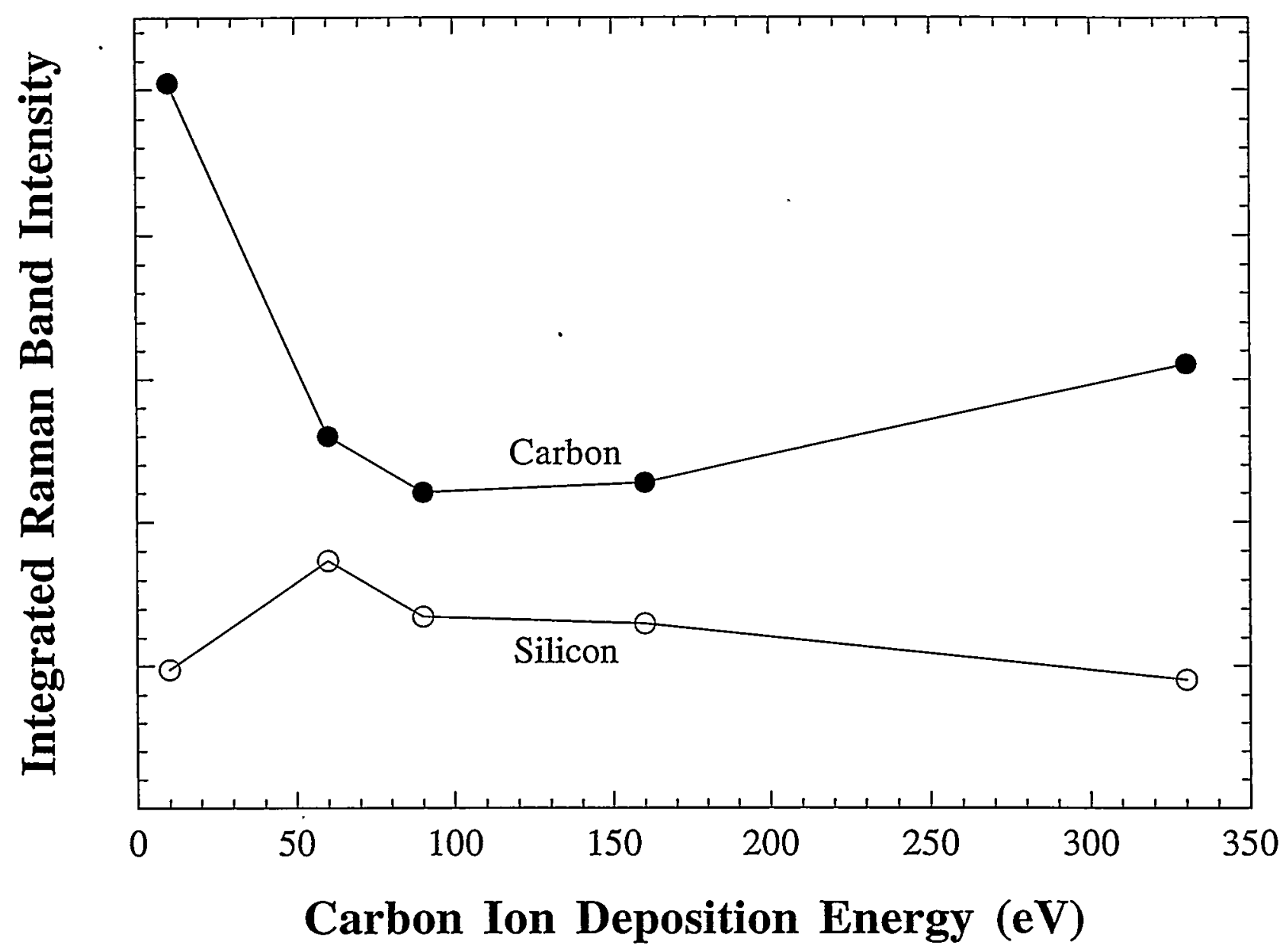

Fig.4 


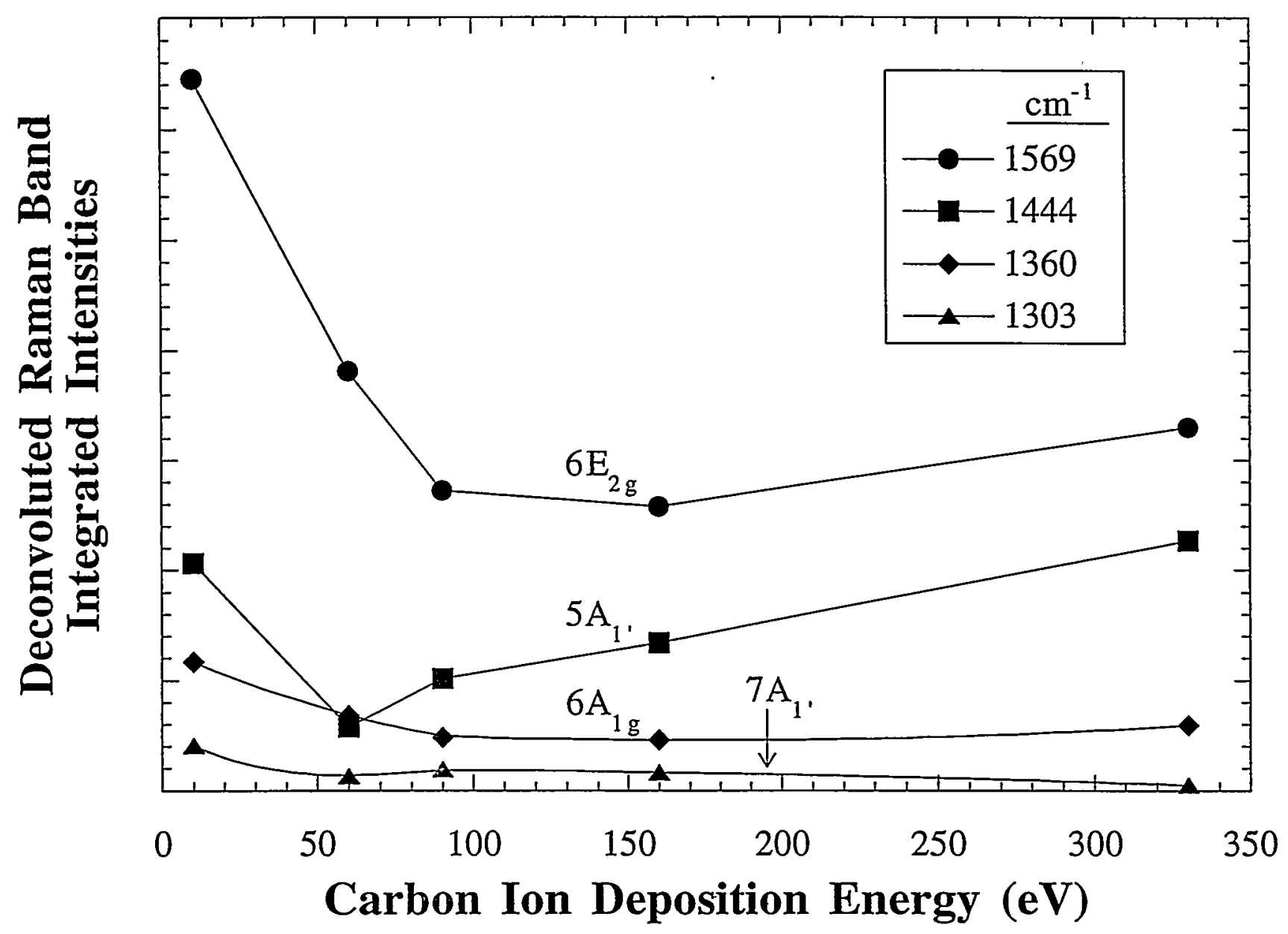

Fig. 5 